\title{
Flare Longest Diameter
}

National Cancer Institute

\section{Source}

National Cancer Institute. Flare Longest Diameter. NCI Thesaurus. Code C112281.

The longest diameter of the area of redness that forms around the site of an antigenic challenge to the skin. 